\title{
A systematic review of nutritional support in haematopoietic stem cell transplantation
}

\author{
M. Crowther ${ }^{1,2}$, D. Culligan ${ }^{2}$, M. Mitchell ${ }^{3}$ and A. Avenell ${ }^{1}$ \\ ${ }^{1}$ Health Services Research Unit, University of Aberdeen, Aberdeen AB25 2ZD, UK and Departments of Haematology ${ }^{2}$ \\ and Dietetics ${ }^{3}$, Aberdeen Royal Infirmary, Aberdeen AB25 2ZD, UK
}

Haematopoietic stem cell transplantation (HSCT) is one of the most physiologically stressful medical treatments performed today ${ }^{(1)}$. Complications include vomiting, diarrhoea, infections, bleeding, mucositis and graft- $v$.-host disease (GvHD), all of which lead to poor nutrition $^{(1)}$. This outcome is combined with possible pre-existing malnutrition from cachexia of malignancy and previous courses of chemotherapy. Studies have demonstrated that patients undergoing HSCT who are underweight have an increased mortality and morbidity. Studies of several nutritional interventions have been undertaken in HSCT. A Cochrane review was produced in $2000^{(2)}$ and new trials have since become available. A new systematic review was therefore performed.

Cochrane methodology for the production of a systematic review was used. Five databases and conference abstracts were searched revealing twenty-nine randomised control trials and eleven non-randomised studies. The most common intervention was glutamine supplementation, either intravenous or oral. Other interventions were parenteral or enteral nutrition and formulations of parenteral nutrition.

Glutamine appeared to have no effect on mortality, time to marrow recovery, diarrhoea and period on total parenteral nutrition (d). Oral glutamine may reduce mucositis (average mucositis score; standard mean difference -0.38 (95\% CI - 0.59, - 0.16)) and GvHD (risk ratio $0.42(95 \%$ CI $0.21,0.85)$ ) while having no effect on length of stay, infections or relapse rate. Intravenous glutamine possibly reduces infections (positive cultures; risk ratio $0.72(95 \%$ CI $0.57,0.91)$ ) and length of stay (mean difference -1.28 (95\% CI $-2.33,-0.23) \mathrm{d}$ ), but has no effect on mucositis and GvHD and may increase relapse rate (risk ratio 2.91 (95\% CI 1.34, 6.29)).

Parenteral nutrition may reduce the long-term mortality (risk ratio 0.78 (95\% CI 0.63, 0.98) and relapses (risk ratio 0.63 (95\% CI 0.44 , $0.90)$ ) but possibly increases infection (positive cultures; risk ratio 1.78 (95\% CI 1.34, 2.36)).

However, the findings should be interpreted with caution as the quality of the trials was generally poor, with infrequent allocation concealment, blinding and intention-to-treat analysis. There was also considerable heterogeneity between the trials in terms of transplant type, disease, age and dosing schedules. Sensitivity and subgroup analysis demonstrated that the results are influenced by including nonrandomised studies and studies without intention-to-treat analysis.

1. Craddock C \& Chakraverty R (2005) In Postgraduate Haematology, 5th ed., pp. 419-435 [AV Hoffbrand, D Catovsky and EGD Tuddenham, editors]. Oxford, UK: Blackwell.

2. Murray SM \& Pindoria S (2007) Cochrane Database of Systematic Reviews, issue 4, CD002920, Chichester, West Sussex: John Wiley and Sons Ltd. 